\title{
Priming negatively affects feeding behaviour and aphid biomass of Rhopalosiphum padi on barley
}

\author{
Gwendolin Wehner $^{1}$ (D) Adam Schikora $^{2}$ D $\cdot$ Frank Ordon $^{1}\left(\mathbb{D} \cdot\right.$ Torsten Will $^{1}$ D
}

Received: 17 February 2020 / Revised: 7 December 2020 / Accepted: 5 January 2021 / Published online: 9 February 2021

(c) The Author(s) 2021

\begin{abstract}
Plants have developed numerous strategies for responding to abiotic and biotic stresses. In particular, the microbiota surrounding plants may have a positive effect on plant stress responses. One is the reaction to rhizobacteria, which can lead to induced systemic resistance. Gram-negative soil bacteria that produce $N$-acyl homoserine lactones (AHL), for example, Ensifer meliloti, induce a primed state in plants that is part of the inducible resistance phenomenon. Observing Rhopalosiphum padi feeding behaviour on a priming sensitive barley genotype, treated with the AHL-producing E. meliloti strain exp $R+c h$, using electrical penetration graph technique showed decreased ingestion of food. Aphids appear to overcome this effect within the eight-hour observation period, possibly explaining the absence of differences of reproduction. Reproduction was observed for a period of 14 days on primed and control-treated plants. Long-term observations over a period of 40 days after aphid infestation showed a lower aphid biomass in contrast to a control group, interpreted as delayed population growth, and an increase in the biomass of barley plants. Priming-related genotypic effects of the defence response to aphids were observed, with no beneficial effects on the plant genotype when its sensitivity to priming was low. Previously, an AHL-priming sensitive barley genotype showed enhanced resistance against fungi when primed with the $\exp R+c h$ strain of E. meliloti. The present study reports the same effect against $R$. padi. These findings suggest that sensitivity to AHL-priming may represent a new approach for plant breeding, targeting multiple pests in parallel by induced plant resistance.
\end{abstract}

Keywords Ensifer meliloti $\cdot$ AHL $\cdot$ Morex $\cdot$ Electrical penetration graph technique $\cdot$ Plant biomass $\cdot$ Plant protection

\section{Key message}

Communicated by Lara Jaber.

Supplementary Information The online version contains supplementary material available at (https://doi.org/10.1007/ s10340-021-01329-8)

\section{Gwendolin Wehner}

gwendolin.wehner@julius-kuehn.de

1 Institute for Resistance Research and Stress Tolerance, Julius Kühn Institute (JKI) - Federal Research Centre for Cultivated Plants, Erwin-Baur-Strasse 27, 06484 Quedlinburg, Germany

2 Institute for Epidemiology and Pathogen Diagnostics, Julius Kühn Institute (JKI) - Federal Research Centre for Cultivated Plants, Messeweg 11/12, 38104 Braunschweig, Germany
- The use of insecticides is reduced for ecological reasons and societal demands. It is therefore necessary to focus on other IPM approaches where priming is a state-of-theart technology for pest control.

- Barley can be primed by AHL-producing Ensifer meliloti resulting in enhanced resistance towards Rhopalosiphum padi.

- Barley genotypes show different sensitivities to AHLpriming.

- AHL priming may involve similar plant-related defence mechanisms against fungal and insect pests. 


\section{Introduction}

Sieve elements (SEs) are part of the phloem and form nutrient transportation conduits inside plants. To access this nutrient source, aphids penetrate plant SEs with their stylet. The initial phase of SE penetration is usually associated with watery saliva secretion into the cell lumen before ingestion starts (Prado and Tjallingii 1994). Effectors are part of the saliva (Elzinga and Jander 2016), and by counteracting plant defences, aphids are able to establish a long-term feeding site. The characteristics of aphid behaviour in such a compatible aphid-plant interaction can be displayed with the electrical penetration graph (EPG) technique, which enables the observation of aphid feeding behaviour. Inside SEs, short periods of watery saliva secretion, followed by long-lasting ingestion periods, are typical for compatible interactions, while an incompatible interaction is characterized by long periods of saliva secretion into SEs and only short subsequent ingestion periods or no ingestion period (e.g. Garzo et al. 2017). During the secretion of saliva into SEs, viruses may be transmitted from a virus-infected aphid to the host plant (Powell et al. 1995). For this reason, the aphid Rhopalosiphum padi (L.) (Hemiptera, Aphididae) represents a serious pest in barley (Hordeum vulgare L.). R. padi damages host plants by withdrawing nutrients and it is the principal vector of Barley yellow dwarf virus (BYDV). In cereals, such as barley and wheat, insecticides are generally used to control $R$. padi infestation and BYDV spread.

In addition to their target organism, insecticides may harm nontarget organisms such as beneficial insects. Insecticides are being banned in parts of the world for that reason, e.g. in the European Union. Due to increased arthropod resistance, insecticide application has become more problematic and alternative methods for controlling insect pests in agricultural must be developed (Sternberg and Thomas 2017). The cultivation of resistant varieties as a measure of integrated pest management (IPM) is an important strategy (Stenberg 2017). Other approaches that promote plant resistance, for example, include microorganisms or biostimulants and are currently under development (Mhlongo et al. 2018).

In recent years, the use of biofertilization to cope with unfavourable climatic conditions and pathogen attacks has become increasingly popular (García-Fraile et al. 2015). Different strategies using various priming inducers, i.e. rhizobia and fungi, have been developed, as well as strategies using their metabolic products, e.g. bacterial liposaccharides (Newman et al. 2007). Pseudomonas fluorescens produces phenazine-1-carboxylic acid (Lohitha et al. 2016); root-colonizing bacteria produce the natural compound $\beta$-aminobutyric acid (Conrath et al. 2002;
Mauch-Mani and Mauch 2005; Zimmerli et al. 2000); and rhizobacteria produce $\mathrm{N}$-acyl homoserine lactone (AHL) (Mathesius et al. 2003; Parsek and Greenberg 2000; Schikora et al. 2011; Schuhegger et al. 2006; Viswanath et al. 2016).The use of living organisms to support naturally occurring resistance mechanisms is preferable to the application of synthetic compounds because of its potentially longer-lasting effects, although no data exist so far. Priming induces an alerted state in plants (Schikora et al. 2016; Viswanath et al. 2016) that results in stronger defence responses when the plants are infected by pathogens (Mauch-Mani et al. 2017). The priming-induced systemic resistance (ISR) can influence direct antagonistic interactions (e.g. via antibiotic production) with pathogens. In particular, AHL priming is a well-analysed system (Schenk and Schikora 2015). The beneficial effects of AHL priming on ISR mechanisms protecting plants against a variety of e.g. fungal pathogens have already been demonstrated in crop plants such as barley and wheat (Hernández-Reyes et al. 2014; Schikora et al. 2011; Shrestha et al. 2019; Wehner et al. 2019). In this system, priming is triggered via the AHL $N$-(3-oxotetradecanoyl)- $L$-homoserine lactone (oxo-C14-HSL), produced as a chemical component for quorum sensing by E. meliloti, a naturally occurring gram-negative soil bacterium that is located in the rhizosphere (Zarkani et al. 2013). Studying AHL priming as a model in the laboratory, different authors have tested resistance towards numerous pathogens, e.g. Alternaria alternata (Schuhegger et al. 2006), Pythium aphanidermatum, Botrytis cinerea (Pang et al. 2009), Blumeria graminis, Puccinia hordei, Puccinia graminis and Phytophthora spp. (Hernández-Reyes et al. 2014; Shrestha et al. 2019; Wehner et al. 2019) as well as Pseudomonas syringae (Shrestha et al. 2020). Wehner and colleagues (2019) have observed that barley genotypes can differ in their sensitivity to AHL when treated with E. meliloti, indicated by the intensity of the resistance response to $P$. hordei. Regarding AHL perception by plants as well as subsequent signal transduction, which involves the production of salicylic acid and the activation of several defence genes like WRKY22, WRKY29 and GST6 (Shrestha et al. 2020), not much is known so far (Shrestha and Schikora 2020). In addition to ISR induction, root growth promotion was observed in Arabidopsis thaliana primed with AHL (Liu et al. 2012; Zhao et al. 2015), whereas a strong increase in root growth and plant biomass was observed when A. thaliana was treated with oxo-C14-HSL, produced by E. meliloti (Shrista et al. 2020).

Based on the above-mentioned studies that report an increase in resistance to pathogenic fungi after AHL-based priming by rhizobacteria in barley, and a report on the increase in plant resistance to aphids (incompatible reaction) in organic broad beans after treatment with the plant growth 
promoting bacterium B. velezensis (Serteyn et al. 2020), we assume that $E$. meliloti-associated AHL priming could be a strategy to increase resistance of barley to $R$. padi. This could be indicated by observing an aphid feeding behaviour similar to an incompatible reaction. Additional parameters influenced by food intake (e.g. reproduction) are also considered to complement observations of feeding behaviour. Furthermore, we have measured plant biomass production to verify the effect of priming on the plant side. To investigate a possible influence of genotype-specific sensitivity to AHL, as observed by Wehner et al (2019), we recorded two genotypes with high and low AHL sensitivity.

\section{Materials and methods}

\section{Plant cultivation and priming}

Two spring barley (Hordeum vulgare L.) lines, i.e. the cultivar Morex and the accession BCC1415 (Genobar Barley Core Collection) (Pasam et al. 2012), differed in their response to leaf rust resistance under AHL-priming, were used. Morex showed a higher sensitivity to AHL-priming, indicated by a stronger priming capacity (Wehner et al. 2019) and was thus selected together with BCC1415 for a detailed analysis of the impact of priming with the AHLproducing rhizobacterium E. meliloti $\exp R^{+} \mathrm{ch}$ on the feeding behaviour and reproduction of $R$. padi. Two days after germination, three seedlings were planted in $60 \mathrm{~mm}$ pots filled with standardised soil (Fruhstorfer type $\mathrm{T}$ ) and grown under controlled conditions (day/night regime of $16: 8 \mathrm{~h}$, temperature of $20-22{ }^{\circ} \mathrm{C}$ day/17-19 ${ }^{\circ} \mathrm{C}$ night, RH $60 \%$ ). Plants were inoculated two, eight and 14 days after planting (dap) as described in Wehner et al. (2019) by soil application of $3.5 \mathrm{ml}$ of bacteria suspension $\left(10^{8} \mathrm{CFU} / \mathrm{ml}\right)$ from one of the two E. meliloti strains. The E. meliloti exp $R^{+} c h$ line produced the AHL $N$-(3-oxotetradecanoyl)- $L$-homoserine lactone (oxo-C14-HSL), while E. meliloti attM, carrying the lactonase gene attM from Agrobacterium tumefaciens on the plasmid pBBR2-attM, resulting in a repression of AHL accumulation, was used as a negative control (Zarkani et al., 2013). The experimental design was a split-plot design containing subplots for each bacterial inoculation variant and 16 replications of each variant. The whole experiment was repeated three times in a controlled environment under the conditions described above.

\section{Aphids}

Rhopalosiphum padi biotype R07 (JKI) individuals were reared on 3- to 4-week-old Hordeum vulgare cv. Rubina (Zadoks scale 11-12) in plastic cages with gauze-covered windows in a greenhouse under controlled environmental conditions (day/night regime of $16: 8 \mathrm{~h}$, at a temperature of $22{ }^{\circ} \mathrm{C}-24{ }^{\circ} \mathrm{C}$ and a relative humidity of $60 \%$ ) (Lantos et al. 2019). The plants were replaced at 14 day intervals.

\section{EPG monitoring}

Two eight-channel 'Giga 8' DC amplifiers (EPG-Systems, Wageningen, NL) were used together with the software 'Stylet $+\mathrm{d}$ ' (EPG-Systems) for data recording. Apterous female adults of $R$. padi were randomly collected from plants inside the rearing cages. Next, they were immobilized by vacuum, and a 2-cm-long gold wire (18 $\mu \mathrm{m}$ diameter) was attached to the dorsal abdomen using water-soluble silver glue (EPG Systems). Subsequently, the aphids were connected to a preamplifier (Tjallingii 1985). Before the aphids were placed on the lower side of the latest mature leaf, they were starved for $60 \mathrm{~min}$. A Faraday cage surrounded the experimental set-up to prevent interference from electrical noise signals. Data recording for a total period of $8 \mathrm{~h}$ started immediately after each of the aphids was placed on a leaf of an individual plant. EPG data from aphids that disconnected or died during data recording were excluded from further analysis. Ten to 13 successful replications (individual aphids) were conducted for each treatment. Experiments were conducted under standardized conditions at room temperature $\left(18-22{ }^{\circ} \mathrm{C}\right)$ under artificial illumination within four days. All variants were measured in independent experimental runs.

The EPG recordings were analysed according to Tjallingii and Esch (1993). The different waveforms related to different behaviours, i.e. pathway activities (C), water uptake from xylem $(\mathrm{G})$, penetration difficulties $(\mathrm{F})$, salivation into the SE (E1) and the ingestion of SE sap (E2), were detected during the analysis. Furthermore, periods of nonpenetration (NP) were detected. 'Stylet $+\mathrm{a}$ ' software (EPG Systems) was used to analyse the duration as well as the frequency of occurrence of the respective behaviour patterns. An Excel-VBA Macro (Schliephake et al. 2013) was used for the calculation of the durations of the respective EPG parameters (Table S1), whereas durations were summed at hourly intervals over the recording time of $8 \mathrm{~h}$ as well as for the total period.

\section{Aphid reproduction and biomass}

Rhopalosiphum padi reproduction was determined on the two barley genotypes, i.e. Morex and BCC1415, in the three-leaf stage (16 dap, Zadoks scale 13) and for the two priming treatments, i.e. E. meliloti strain $\exp R+c h$ as the priming treatment and $a t t M$ as the control treatment ( $n=12$ for each genotype and treatment), by counting the daily production of offspring using clip cages (Abdellatef et al. 2015). Nymphs were removed from the plants every day, and the total number of nymphs was summed over a 
period of 7 days (the time when aphids reach their maximal reproduction rate, nymphs/day; cf Abdellatef et al. 2015) and 14 days.

To observe the long-term reproduction of $R$. padi on the respective barley genotypes and treatments, five adults were placed on each plant $(n=12$ for each genotype and treatment; Zadoks scale 12-13), and the plants were covered with crispack bags. The aphids were allowed to reproduce for 40 days. At this time, the aphids started to walk off of the plants due to the high population density. Aphids were completely removed from the plants (56 dap, Zadoks scale 29) by washing the plants gently with $70 \%$ ethanol. The aphids were collected on filter paper and dried at room temperature for $2 \mathrm{~h}$. Insect biomass was determined by using a fine-scale balance (Kern ABS 120-4, KERN \& Sohn GmbH, Balingen-Frommern, Germany). For both approaches, age-synchronized 8-day-old adult apterous aphids were used, and both experiments were conducted under controlled environmental conditions as previously described for aphid rearing.

\section{Plant biomass}

Plants from the aphid mass reproduction experiment were cut directly above the ground and used to analyse the plant biomass of the control (E. meliloti attM) and primed plants (E. meliloti $\exp R+c h$ ). Leaf material from plants of the two lines and from both inoculation variants (with and without aphid infestation) was collected from individual plants, dried at $105{ }^{\circ} \mathrm{C}$ for $24 \mathrm{~h}$ and weighed (Kern ABS 120-4).

\section{Statistical analysis}

Statistical analysis of the EPG parameter data obtained by using the Excel-VBA Macro (Schliephake et al. 2013) was performed using the Mann-Whitney U test. Outliers in the aphid reproduction experiment and plant biomass data were identified using Huber M-estimation (Huber and Ronchetti 2009). Outliers were defined as values that were two times the spread from the centre and were subsequently excluded from further analysis. The normal distribution of the data was checked (Shapiro-Wilk W test), and further analyses of the differences in reproduction and biomass were performed by t tests $(\alpha=0.95)$. Statistical analysis was conducted using JMP v 12.0.1 software (SAS Institute Inc., Cary, USA).

\section{Results}

\section{Aphid behaviour}

To test for differences in the susceptibility of both barley genotypes as well as for the effect of AHL on the susceptibility of the two barley genotypes at an early developmental stage, we observed the feeding behaviour of $R$. padi using the EPG technique.

A comparison of the differences in $R$. padi feeding behaviour between the barley genotypes with low (BCC1415) and high AHL-priming sensitivity (Morex) in the control group

Table 1 Comparison of EPG parameters of $R$. padi on control-treated plants of barley cultivars BCC1415 and Morex over an observation period of $8 \mathrm{~h}$

\begin{tabular}{|c|c|c|c|c|c|c|c|}
\hline \multirow[b]{2}{*}{ Parameter } & \multicolumn{3}{|l|}{ BCC 1415} & \multicolumn{3}{|l|}{ Morex } & \multirow{2}{*}{$\begin{array}{l}\text { Mann- } \\
\text { Whitney } \mathrm{U} \\
\text { test } \\
p \text { value }\end{array}$} \\
\hline & Mean duration $[\mathrm{s}]$ & Median duration $[\mathrm{s}]$ & Std. error & Mean duration $[\mathrm{s}]$ & Median duration $[\mathrm{s}]$ & Std.-error & \\
\hline s_C & 8264.11 & 8587.53 & 1362.91 & 4391.17 & 2388.13 & 1419.91 & 0.0321 \\
\hline s_E1 & 182.53 & 30.13 & 126.81 & 555.72 & 274.84 & 291.8 & 0.0155 \\
\hline s_E2 & 7648.59 & 1522.41 & 2979.06 & $19,187.09$ & $22,482.33$ & 2469.63 & 0.0155 \\
\hline s_F & 3347.6 & 610.21 & 1410.36 & 142.49 & 6.31 & 83.24 & 0.041 \\
\hline s_G & 6202.89 & 4509.4 & 1926.46 & 2363.25 & 0 & 1056.9 & ns \\
\hline s_Np & 3154.28 & 2335.11 & 697.7 & 2160.3 & 1383.87 & 608.94 & ns \\
\hline s_Pr & $25,645.72$ & $26,464.89$ & 697.7 & $26,639.7$ & $27,416.14$ & 608.94 & ns \\
\hline s_sE2 & 7576.58 & 1090.35 & 2994.8 & $19,086.05$ & $22,482.33$ & 2498.91 & 0.0151 \\
\hline $\mathrm{t} \_1 \mathrm{E}$ & $18,203.34$ & $24,496.31$ & 3044.75 & 6037.43 & 3273.49 & 2163.5 & 0.0076 \\
\hline t_1E1_1E2 & $11,412.55$ & 620.55 & 4000.77 & 409.63 & 26.19 & 321.68 & 0.0134 \\
\hline $\mathrm{T}$ & $19,875.77$ & $25,286.6$ & 3002.63 & 8674.73 & 5994.21 & 2387.71 & 0.0192 \\
\hline t_1E2 & $18,337.07$ & $24,510.68$ & 3004.45 & 6447.06 & 4377.32 & 2132.71 & 0.0076 \\
\hline $\mathrm{t} \_1 \mathrm{G}$ & $11,206.23$ & $10,830.88$ & 2684.78 & $18,151.43$ & $27,183.26$ & 4086.42 & ns \\
\hline $\mathrm{t} \_1 \mathrm{Pr}$ & 1347.66 & 725.66 & 410.89 & 1039.22 & 496.02 & 307.07 & ns \\
\hline
\end{tabular}


(E. meliloti attM) indicated significant differences $(p<0.05)$ for several EPG parameters (Table 1). While the summed duration of pathway activities (s_C) was reduced, the summed durations of ingestion (s_E2) and sustained ingestion (s_sE2) were longer for the genotype Morex than for the genotype BCC1415 (Table 1). No difference was detected for the time until first probing ( $\left.t \_1 P r\right)$, but earlier phloem contact ( $\left.t \_1 E\right)$, earlier ingestion after a first SE contact (t_1E1_1E2) and a reduced time to first sustained ingestion (t_1E1_1 sE2) were observed for aphids feeding on Morex. No differences were observed between the tested genotypes for the summed whole probing time (s_Pr) or the summed duration of xylem contact (s_G).

To study the impact of AHL-priming on the feeding behaviour of R. padi, E. meliloti expR + ch-treated plants were compared to plants of the same genotype that were treated with the E. meliloti attM strain, unable to accumulate AHL. The data revealed (Fig. 1, Table 2) that the time without plant contact (s_NP, Fig. 1f,n), summed probing time (s_Pr, Fig. 1g,o), summed duration of penetration problems (s_F, Fig. 1d,l) and summed duration of pathway activities of the stylet within the plant tissue (s_C, Fig. 1a,i) were not affected by either treatment of the respective plant genotypes. For the genotype Morex, the AHL treatment caused a significant $(p<0.05)$ decrease in ingestion (s_E2, Fig. 1k) and sustained ingestion (s_sE2, Fig. 1p) starting in the third hour of observation and increased xylem contact (s_G, Fig. 1m) after the fifth hour of observation (Fig. 1). Aphids on $\exp R+c h$-treated plants of the genotype Morex also showed a delayed first SE contact (t_1E) and later sustained ingestion after the first SE contact (t_1E1_1sE2) within the observation period (Table 2). Parameter s_E1 (Fig. 1j,1; Table 2) and parameters t_1E, t_1E1_1E2, t_1G and $t \_1$ Pr were not affected as a consequence of the AHL treatment (Table 2).

For the genotype BCC1415, priming by E. meliloti $\exp R^{+} c h$ led to a significantly higher rate of secretion of watery saliva into SEs (s_E1, Fig. 1b) after a 5-h plant-aphid

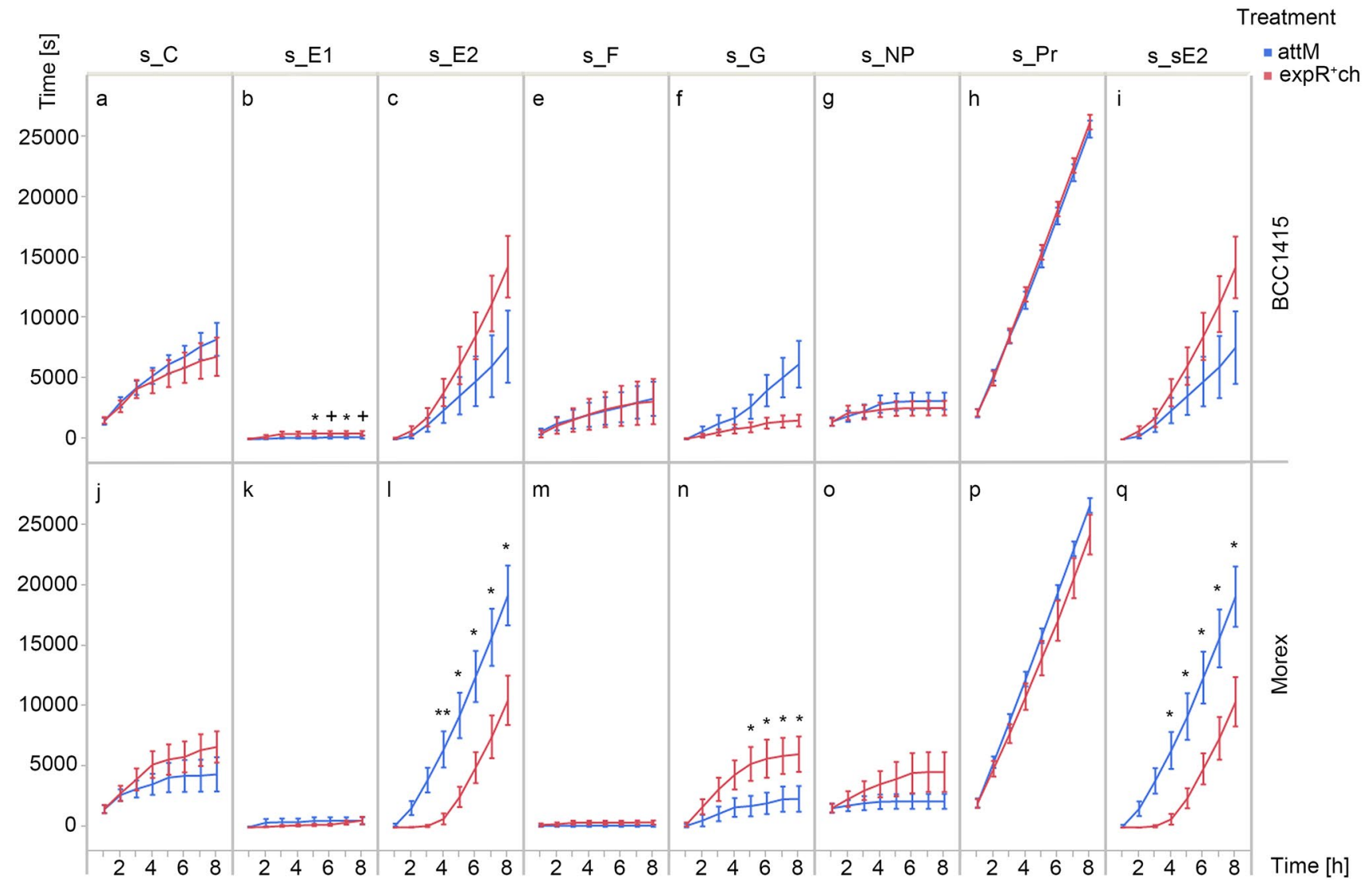

Fig. 1 EPG parameters calculated from observation of $R$. padi on control and primed barley genotypes BCC1415 a-i and Morex jq. Within a probe (Pr), the electrical signal recorded from an aphid shows the behaviour-related waveforms: pathway activities $(\mathrm{C})$, water uptake from xylem $(\mathrm{G})$, penetration difficulties $(\mathrm{F})$, salivation into SEs (E1) and ingestion of SE sap (E2). Durations of respective waveforms were summed up at hourly intervals for the recording period of
$8 \mathrm{~h}$ for subsequent calculation of the EPG parameters s_C a, i, s_E1 $\mathbf{b}, \mathbf{j}$, s_E2 c, k, s_F d, l, s_G e, m, s_NP f, n, s_Pr $\mathbf{g}, \mathbf{o}$ and s_sE2 $\mathbf{h}$, p. Mean values (error bars indicate standard error) are calculated and the nonparametric Mann-Whitney U test was used for statistical analysis, whereas asterisks indicate a significant difference with $p<0.05$ and a plus indicates a tendency with $p=0.05-0.07$ 
Table 2 Comparison of EPG parameters of R. padi on control-treated and primed plants within the barley cultivars BCC1415 and Morex over an observation period of $8 \mathrm{~h}$

\begin{tabular}{|c|c|c|c|c|c|c|c|c|}
\hline \multirow[t]{2}{*}{ Genotype } & \multirow[t]{2}{*}{ Variable } & \multicolumn{3}{|l|}{$a t t M$} & \multicolumn{3}{|l|}{$\exp R+c h$} & \multirow{2}{*}{$\begin{array}{l}\text { Mann- } \\
\text { Whitney } \mathrm{U} \\
\text { test } \\
p \text { value }\end{array}$} \\
\hline & & Mean duration (s) & Median duration (s) & Std.-error & Mean duration (s) & Median duration (s) & Std.-error & \\
\hline \multirow[t]{14}{*}{ BCC1415 } & s_C & 8264.11 & 8587.53 & 1362.91 & 6823.61 & 6414.24 & 1580.72 & ns \\
\hline & s_E1 & 182.53 & 30.13 & 126.81 & 483.67 & 114.35 & 192.81 & ns \\
\hline & s_E2 & 7648.59 & 1522.41 & 2979.06 & $14,264.02$ & $18,484.97$ & 2545.63 & ns \\
\hline & S_F & 3347.6 & 610.21 & 1410.36 & 3113.33 & 0 & 1873.27 & ns \\
\hline & s_G & 6202.89 & 4509.4 & 1926.46 & 1545.82 & 992.49 & 490.35 & ns \\
\hline & s_Np & 3154.28 & 2335.11 & 697.7 & 2569.55 & 2134.73 & 605.03 & ns \\
\hline & s_Pr & $25,645.72$ & $26,464.89$ & 697.7 & $26,230.45$ & $26,665.27$ & 605.03 & ns \\
\hline & s_sE2 & 7576.58 & 1090.35 & 2994.8 & $14,215.67$ & $18,484.97$ & 2548.51 & $\mathrm{~ns}$ \\
\hline & $\mathrm{t} \_1 \mathrm{E}$ & $18,203.34$ & $24,496.31$ & 3044.75 & $11,888.71$ & 9519.45 & 2502.59 & ns \\
\hline & $\mathrm{t} \_1 \mathrm{E} 1 \_1 \mathrm{E} 2$ & $11,412.55$ & 620.55 & 4000.77 & 5148.8 & 50.45 & 2821.39 & ns \\
\hline & t_1E1_1sE2 & $19,875.77$ & $25,286.6$ & 3002.63 & $13,831.82$ & 9760.14 & 2506.03 & ns \\
\hline & $\mathrm{t} \_1 \mathrm{E} 2$ & $18,337.07$ & $24,510.68$ & 3004.45 & $12,789.55$ & 9567.72 & 2553.53 & ns \\
\hline & $\mathrm{t} \_1 \mathrm{G}$ & $11,206.23$ & $10,830.88$ & 2684.78 & $15,348.43$ & $13,143.01$ & 2979.61 & ns \\
\hline & $\mathrm{t} \_1 \mathrm{Pr}$ & 1347.66 & 725.66 & 410.89 & 620.44 & 482.3 & 150.83 & ns \\
\hline \multirow[t]{14}{*}{ Morex } & s_C & 4391.17 & 2388.13 & 1419.91 & 6659.93 & 5369.2 & 1287.64 & $\mathrm{~ns}$ \\
\hline & s_E1 & 555.72 & 274.84 & 291.8 & 572.73 & 182.61 & 311.13 & ns \\
\hline & s_E2 & $19,187.09$ & $22,482.33$ & 2469.63 & $10,516.26$ & $12,900.32$ & 2040.24 & 0.0173 \\
\hline & s_F & 142.49 & 6.31 & 83.24 & 409 & 46.89 & 176.17 & $\mathrm{~ns}$ \\
\hline & s_G & 2363.25 & 0 & 1056.9 & 6059.04 & 5397.75 & 1463.49 & 0.0407 \\
\hline & s_Np & 2160.3 & 1383.87 & 608.94 & 4583.05 & 3369.06 & 1648.3 & ns \\
\hline & s_Pr & $26,639.7$ & $27,416.14$ & 608.94 & $24,216.95$ & $25,430.95$ & 1648.3 & ns \\
\hline & s_sE2 & $19,086.05$ & $22,482.33$ & 2498.91 & $10,386.72$ & $12,803.97$ & 2040.8 & 0.014 \\
\hline & $\mathrm{t} \_1 \mathrm{E}$ & 6037.43 & 3273.49 & 2163.5 & 8997.82 & 7341.02 & 2388.89 & ns \\
\hline & t_1E1_1E2 & 409.63 & 26.19 & 321.68 & 5457.85 & 779.67 & 2228.1 & ns \\
\hline & t_1E1_1sE2 & 8674.73 & 5994.21 & 2387.71 & $16,727.97$ & $14,950.42$ & 1905.15 & 0.014 \\
\hline & $\mathrm{t} \_1 \mathrm{E} 2$ & 6447.06 & 4377.32 & 2132.71 & $14,455.67$ & $13,693.99$ & 2086.71 & 0.0091 \\
\hline & $\mathrm{t} \_1 \mathrm{G}$ & $18,151.43$ & $27,183.26$ & 4086.42 & 7975.54 & 4342 & 2611.92 & $\mathrm{~ns}$ \\
\hline & $\mathrm{t} \_1 P r$ & 1039.22 & 496.02 & 307.07 & 1654.45 & 554.93 & 834.45 & ns \\
\hline
\end{tabular}

interaction period (Fig. 1). The parameters s_E2, s_sE2 and s_G (Fig. 1c,e,h) showed the opposite effect of the responses observed in Morex to E. meliloti strain $\exp R+c h$ application, but in total, none of the parameters was significantly different between the treatments during the observation period (Fig. 1a-h, Table 2).

Examining the significantly different EPG parameter summed duration of sustained ingestion (s_sE2) for the two treatments on Morex (Fig. 1p) suggests two different regions in the graph progression. We separated the observation period into two parts: $1-5 \mathrm{~h}$ and $5-8 \mathrm{~h}$, based on the equation calculated when results from the given period were plotted as a function of time (Table 3). While both time periods for the $a t t M$-treated plants had linear trend lines, the equation for the $\exp R+c h$-treated plants showed a quadratic function for the first period (1-5 h) and a linear function for
Table 3 Line equations derived from curve progression of s_sE2 of control-treated and $E$. meliloti $\exp R+$ ch-treated barley genotype Morex

\begin{tabular}{llll}
\hline Equation No & Time period $(\mathrm{h})$ & Line equation & $\begin{array}{l}\text { Coefficient of } \\
\text { determination }\end{array}$ \\
\hline 1 & $1-5$ & $y($ attM $)=-2674+2295 * x$ & 0.99 \\
2 & $1-5$ & $y(\exp R+c h)=1000-1182 * x+292.9 * x^{2}$ & 0.97 \\
3 & $5-8$ & $y($ attM $)=-7288+3301 * x$ & 1.00 \\
4 & $5-8$ & $y(\exp R+c h)=-10843+2649 * x$ & 1.00 \\
\hline
\end{tabular}


the second period $(5-8 \mathrm{~h})$ that was nearly parallel to that of the attM-treated plants. This finding suggests the delayed ingestion of SE sap by aphids in the AHL-primed plants.

\section{Aphid reproduction}

We studied the potential short- and long-term effects of AHL-priming on aphid fitness. Therefore, we determined aphid reproduction in intervals of seven and 14 days for individual insects and for a period of 40 days for the whole population (indicated as the aphid population biomass) on individual plants. There were no significant effects $(p>0.05)$ on the mean reproduction of individuals from the treatment with $E$. meliloti strain $\exp R+c h$ on either barley genotype for the two periods (0-7 dai and 7-14 dai, Fig. 2). In contrast, mass reproduction over a period of 40 days showed a significant $(p<0.05)$ reduction in the mean biomass of aphids on the genotype Morex when the plants were treated with $E$. meliloti $\exp R^{+} c h$. There was no difference between the two treatments in genotype BCC1415 (Fig. 3). Hence, AHL-priming had different effects on aphid reproduction in the two genotypes.

\section{Plant biomass}

To validate the hypothesis that plants benefit from AHLpriming, we selected plant biomass as an indicator. For both barley genotypes and both treatments, the plant biomass was significantly $(p<0.05)$ reduced when plants were infested



Fig. 2 Reproduction rate of $R$. padi feeding on control and primed plants. Plants were infested with a single synchronized adult $R$. padi each, two days after the last bacteria application to roots (16 dap). The number of nymphs was counted on a daily basis, summed up for periods of seven successive days, and mean was displayed for two successive weeks from the beginning of reproduction. The $t$ test was used for treatment comparison within a genotype (BCC1415 a and Morex b) for the two time points. No significant difference was detected. Error bars indicate standard error
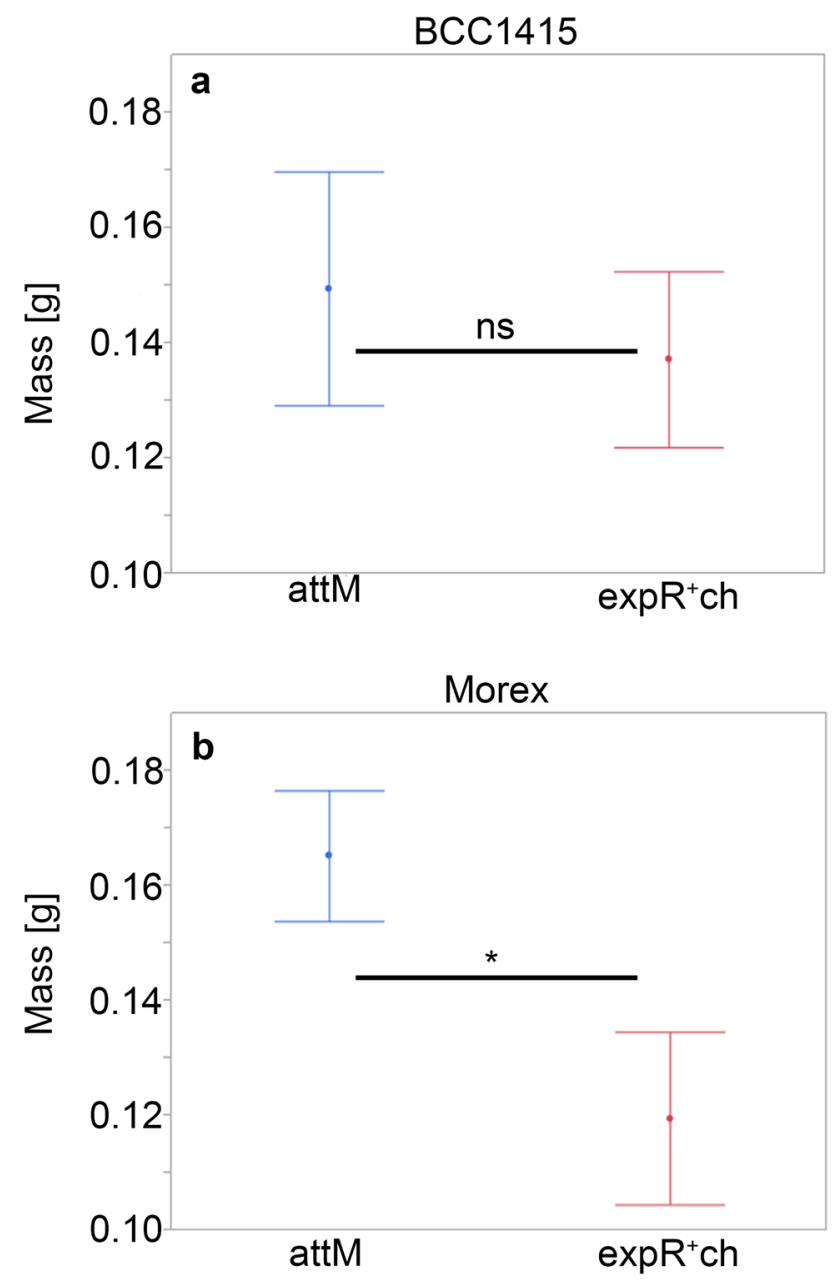

Fig. 3 Buildup of aphid biomass on control and primed plants. Plants were infested with five synchronized adult $R$. padi each, two days after the last bacteria application to roots (16 dap). $R$. padi biomass was measured 40 days after infestation (dai), during which aphids were allowed to reproduce freely, and the $t$ test was used for comparison of the treatments within the respective barley genotypes BCC1415 a and Morex b with $p<0.05$. Error bars indicate standard error

with aphids (Fig. 4). When infested with aphids, the genotype Morex produced significantly $(p<0.05)$ more biomass when it was previously primed with E. meliloti $\exp R^{+} c h$. This effect was absent in BCC1415. In plants not infested by aphids, the $E$. meliloti $\exp ^{+}$ch treatment resulted in a significant $(p<0.05)$ reduction in the mean plant biomass in BCC1415 (Fig. 4). This effect was not observed in Morex.

\section{Discussion}

Research on rhizobacteria-induced plant resistance against insects is mainly focused on resistance to aphids such as $R$. padi (Sugio et al. 2015). In our study, the treatment with an 


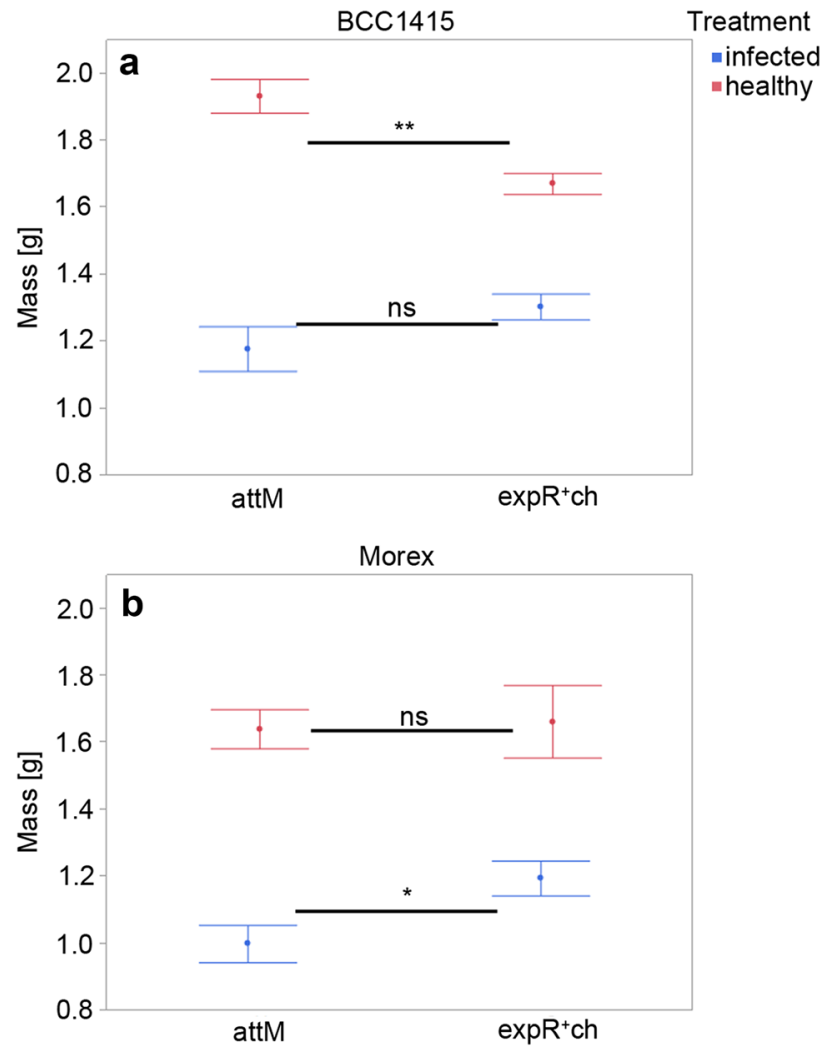

Fig. 4 Plant biomass of control and primed plants with and without aphid infestation. Plant biomass was measured 40 dai with $R$. padi during which aphids were allowed to reproduce freely. The $t$ test was used for comparison of the treatments within the respective barley genotypes BCC1415 a and Morex b with $p<0.05$. Error bars indicate standard error

AHL-producing bacterial strain of E. meliloti caused significant priming effects for the barley genotype Morex, while treatment with an AHL-suppressed strain did not cause priming effects. The observed effects of priming on $R$. padi were reduced ingestion and negative effects on the long-term reproduction (Table 2; Fig. 3). Since a positive response to AHL priming was already described in barley with regard to resistance against leaf rust (Wehner et al. 2019) and powdery mildew (Shrestha et al. 2019), we conclude that AHL priming may negatively affect multiple pests/pathogens in parallel. The observation of negative effects on aphids by priming corresponds to earlier observations on the model plant species Arabidopsis thaliana (Pozo et al. 2008; Van der Ent et al. 2009), where, for example, Bacillus velezensis triggered an ISR against the green peach aphid Myzus persicae (Rashid et al. 2017). By using different mutants, the authors concluded that defence-relevant phytohormones such as salicylic acid (SA) and jasmonic acid (JA) are not part of the priming reaction. Reactive oxygen species whose formation was significantly induced after priming with $B$. velezensis and infestation by M. persicae (Rashid et al. 2017) play an important role in several aphid-plant interactions (e.g. Moloi and van der Westhuizen 2006). Although AHLinduced priming also leads to an accumulation of hydrogen peroxide in plant tissues (Bai et al. 2012), in contrast to priming induced by $B$. velezensis, the plant hormones SA, JA and oxylipin are involved in this mechanism (Schenk and Schikora 2015). Therefore, the priming mechanisms differ significantly in terms of the underlying molecular mechanisms.

However, our data suggest that aphids appear to be confronted with two independent defence mechanisms located in sieve elements (SEs). A short-term mechanism, which affects initial SE penetration and lasts for a period of up to $4 \mathrm{~h}$, resulting in delayed (sustained) ingestion of SE sap from primed plants of the genotype Morex (Fig. 1; Table 2). After this period, the negative effect on ingestion is no longer present. Delayed ingestion may be associated with problems in establishing a long-term feeding site after SE penetration, i.e. because of an intensive defence response located inside SEs (Will et al. 2013). Delayed ingestion is associated with the presence of proteins such as SIEVE ELEMENT-LINING CHAPERONE1 (SLII), a potential structural protein inside the SEs of A. thaliana (Kloth et al. 2017), or the expression of phloem-associated defence genes, as described recently for Hordeum spontaneum (Leybourne et al. 2019). The mechanism of this AHL-induced defence is not known, but due to its short functional period, we assume that salivary effectors could be involved in the suppression of this defence barrier; in contrast, the recently discovered effector $\mathrm{Rp} 1$ from $R$. padi interferes with plant hormone signalling (Escudero-Martinez et al. 2020).

The reduced insect biomass on the primed Morex cultivar after a period of 40 days (Fig. 3) appears to be an indicator of a second mechanism with a negative longterm effect on aphid fitness, i.e. reproduction (Fig. 2). Harming aphid endosymbiotic bacteria, as indicated by the application of antibiotics (Koga et al. 2007), results in reduced aphid growth and/or reproduction. Thus, a nutrient shortage may have led to the delayed accumulation of biomass at the population level. Antimicrobial peptide coding genes, i.e. thionin genes, have been detected in barley and are suggested to be part of the barley defence response against aphids (Escudero-Martinez et al. 2017), as previously shown for other antimicrobial peptides (Campos et al. 2018). The antibacterial activity of thionins could harm the obligate bacterial endosymbionts of aphids, and a similar effect was described for the antimicrobial peptide indolicidin when ingested by the green peach aphid (Le-Feuvre et al. 2007). Similarly, thionins possess antifungal activities, as demonstrated for Fusarium oxysporum in A. thaliana (Epple et al. 1997). However, this explanation is currently speculative and requires further research, but could explain analogous effects of 
priming in the barley variety Morex on leaf rust (Wehner et al. 2019) and R. padi. Wehner et al. (2019) observed that the efficiency of AHL priming differs between barley genotypes. BCC1415 is a genotype with a low priming efficiency, resulting in a low level of induced resistance. When comparing the cultivars Morex and BCC1415, we observed that the aphid behaviour on plants treated with the AHL-producing E. meliloti $\exp R^{+} c h$ strain was different from that of aphids on plants treated with the AHL non-accumulating strain of E. meliloti only in the Morex cultivar (Fig. 1; Table 2). These observations indicate that differences in priming efficiency are also present with regard to resistance against aphids. A similar effect in barley was also shown for the aphid species Sitobion avenae and priming induced by Pseudomonas aeruginosa (Tétard-Jones et al. 2007). The observation that priming might have no effect or a positive effect on aphids, as indicated in our study by behavioural observations on BCC1415, is supported by the findings of Pineda et al. (2010). In that study, plant treatment with the non-pathogenic rhizobacterium Pseudomonas fluorescens led to increased M. persicae body size on A. thaliana.

It appears that the intensity of priming costs may differ between genotypes or can be compensated by some plant genotypes because no negative effect on plant biomass was observed for Morex in the absence of aphids (Fig. 4). Generally, beneficial soil-borne microorganisms are described as being able to induce plant growth when plants are infested with insects (Pineda et al. 2010). However, the mechanisms related to AHL recognition, the subsequent signal transduction and the regulation of defence-related genes in barley are still unknown, and the involvement of antibacterial/antimicrobial peptides is a hypothesis that must be demonstrated in future studies.

The majority of studies in this field have focused on priming effects in A. thaliana. This study, however, aimed to strengthen the translational aspect and increase our knowledge of priming in $H$. vulgare because of its agronomic importance. The differences in the reactions of the two genotypes that were selected for this study indicate that priming is strongly influenced by the genetic background of the respective cultivar. To promote priming as a sustainable and environmentally friendly approach for plant protection, cultivars must be selected according to their response to priming. In this way, the widespread use of priming could provide measurable benefits to farmers.

\section{Author contributions}

GW, TW and FO planned and designed the study. AS provided bacterial strains and protocols for priming. GW and TW performed the experiments, data analysis and wrote the manuscript. All authors read and approved the final manuscript.

Supplementary file 1 (DOCX 13 kb)Acknowledgements The authors would like to thank Christine Hoppe, Kerstin Welzel, Evelyn Betke and Heike Dobrowolski for their excellent technical support.

Funding Open Access funding enabled and organized by Projekt DEAL. Parts of this study are funded by the German Federal Ministry of Education and Research (BMBF) (Grants FKZ 031B0196C and FKZ 031B0196B) in the scope of the Plant2030 perspective.

Data availability All data, which were analysed, are presented in this publication.

Code availability Not applicable.

\section{Compliance with ethical standards}

Conflicts of interest The authors declare that they have no conflict of interest.

Ethical approval This research does not involve the use of animals or humans in experimentation.

Consent to participate Not applicable.

Consent for publication Not applicable.

Open Access This article is licensed under a Creative Commons Attribution 4.0 International License, which permits use, sharing, adaptation, distribution and reproduction in any medium or format, as long as you give appropriate credit to the original author(s) and the source, provide a link to the Creative Commons licence, and indicate if changes were made. The images or other third party material in this article are included in the article's Creative Commons licence, unless indicated otherwise in a credit line to the material. If material is not included in the article's Creative Commons licence and your intended use is not permitted by statutory regulation or exceeds the permitted use, you will need to obtain permission directly from the copyright holder. To view a copy of this licence, visit http://creativecommons.org/licenses/by/4.0/.

\section{References}

Abdellatef E, Will T, Koch A, Imani J, Vilcinskas A, Kogel KH (2015) Silencing the expression of the salivary sheath protein causes transgenerational feeding suppression in the aphid Sitobion avenae. Plant Biotechnol J 13:849-857

Bai X, Todd CD, Desikan R, Yang Y, Hu X (2012) N-3-oxodecanoyl-l-homoserine-lactone activates auxin-induced adventitious root formation via hydrogen peroxide- and nitric oxidedependent cyclic GMP signaling in Mung Bean. Plant Physiol 158:725-736

Campos ML, de Souza CM, de Oliveira KBS, Dias SC, Franco OL (2018) The role of antimicrobial peptides in plant immunity. J Exp Bot 69:4997-5011

Conrath U, Pieterse CM, Mauch-Mani B (2002) Priming in plantpathogen interactions. Trends Plant Sci 7:210-216

Delp G, Gradin T, Ahman I, Jonsson LMV (2009) Microarray analysis of the interaction between the aphid Rhopalosiphum padi 
and host plants reveals both differences and similarities between susceptible and partially resistant barley lines. Mol Gen Genomics 281:233-248

Elzinga DA, Jander G (2013) The role of protein effectors in plantaphid interactions. Curr Opinion Plant Biol 16:451-456

Epple P, Apel K, Bohlmann H (1997) Overexpression of an endogenous thionin enhances resistance of Arabidopsis against Fusarium oxysporum. Plant Cell 9:509-520

Escudero-Martinez C, Rodriguez PA, Liu S, Santos PA, Stephens J, Bos JIB (2020) An aphid effector promotes barley susceptibility through suppression of defence gene expression. J Exp Bot 71:2796-2807

Escudero-Martinez CM, Morris JA, Hedley PE, Bos JIB (2017) Barley transcriptome analyses upon interaction with different aphid species identify thionins contributing to resistance. Plant Cell Environ 40:2628-2643

García-Fraile P, Menéndez E, Rivas R (2015) Role of bacterial biofertilizers in agriculture and forestry. AIMS Bioengineering 2:183-205

Garzo E, Fernández-Pascual M, Morcillo C, Fereres A, Gómez-Guillamón ML, Tjallingii WF (2017) Ultrastructure of compatible and incompatible interactions in phloem sieve elements during the stylet penetration by cotton aphids in melon. Insect Sci 25:631-642

Hernández-Reyes C, Schenk ST, Neumann C, Kogel KH, Schikora A (2014) N-acyl-homoserine lactones-producing bacteria protect plants against plant and human pathogens. Microb Biotechnol 7:580-588

Huber P, Ronchetti E (2009) Robust Statistics, 2nd edn. Wiley, New York

Kloth KJ, Busscher-Lange J, Wiegers GL, Kruijer W, Buijs G, Meyer RC, Albrectsen BR, Bouwmeester HJ, Dicke M (2017) SIEVE ELEMENT-LINING CHAPERONE1 restricts aphid feeding on Arabidopsis during heat stress. Plant Cell 29:2450-2464

Koga R, Tsuchida T, Sahurai M, Fukatsu T (2007) Selective elimination of aphid endosymbionts: effects of antibiotic dose and host genotype, and fitness consequences. FEMS Microbiol Ecol 60:229-239

Lantos E, Schliephake E, Krämer R, Will T, Nothnagel T (2019) Feeding behavior of Myzus persicae on asparagus species susceptible and resistant to Asparagus virus 1. Ent Exp Appl 4:360-369

Le-Feuvre RR, Ramirez CC, Olea N, Meza-Basso L (2007) Effect of the antimicrobial peptide indolicidin on the green peach aphid Myzus persicae (Sulzer). J Applied Entomol 131:71-75

Leybourne DJ, Valentine TA, Robertson JAH, Pérez-Fernández E, Main AM, Karley AJ, Bos JIB (2019) Defence gene expression and phloem quality contribute to mesophyll and phloem resistance to aphids in wild barley. J Exp Bot 70:4011-4026

Liu F, Bian Z, Jia Z, Zhao Q, Song S (2012) The GCR1 and GPA1 participate in promotion of Arabidopsis primary root elongation induced by $\mathrm{N}$-acyl-homoserine lactones, the bacterial quorumsensing signals. Mol Plant-Microbe Interact 25:677-683

Lohitha S, Bhaskara R, Sivaprasad Y, Prathyusha M, Sujitha A, Krishna T (2016) Molecular characterization and antagonistic potential of phenazine-1-carboxylic acid producing Pseudomonas fluorescens isolates from economically important crops in South India. Int J Clin Biol Sci 1:30-40

Mathesius U, Mulders S, Gao M, Teplitski M, Caetano-Anollés G, Rolfe BG, Bauer WD (2003) Extensive and specific responses of a eukaryote to bacterial quorum-sensing signals. PNAS 100:1444-1449

Mauch-Mani B, Mauch F (2005) The role of abscisic acid in plantpathogen interactions. Curr Opin Plant Biol 8:409-414

Mauch-Mani B, Baccelli I, Luna E, Flors V (2017) Defense priming: an adaptive part of induced resistance. Annu Rev Plant Biol 68:485-512
Mhlongo MI, Piater LA, Madala NE, Labuschagne N, Dubery IA (2018) The chemistry of plant-microbe interactions in the rhizosphere and the potential for metabolomics to reveal signaling related to defense priming and induced systemic resistance. Front Plant Sci 9:112

Moloi MJ, van der Westhuizen A (2006) The reactive oxygen species are involved in resistance response of wheat to the Russian wheat aphid. J Plant Physiol 163:1118-1125

Newman M-A, Dow JM, Molinaro A, Parrilli M (2007) Invited review: priming, induction and modulation of plant defence responses by bacterial lipopolysaccharides. J Endotoxin Res 13:69-84

Ni XZ, Quisenberry SS, Heng-Moss T, Markwell J, Sarath G, Klucas R, Baxendale F (2001) Oxidative responses of resistant and susceptible cereal leaves to symptomatic and nonsymptomatic cereal aphid (Hemiptera: Aphididae) feeding. J Econ Entomol 94:743-751

Pang Y, Liu X, Ma Y, Chernin L, Berg G, Gao K (2009) Induction of systemic resistance, root colonisation and biocontrol activities of the rhizospheric strain of Serratia plymuthica are dependent on N-acyl homoserine lactones. Eur J Plant Pathol 124:261-268

Parsek MR, Greenberg EP (2000) Acyl-homoserine lactone quorum sensing in gram-negative bacteria: a signaling mechanism involved in associations with higher organisms. PNAS 97:8789-8793

Pasam RK, Sharma R, Malosetti M, van Eeuwijk FA, Haseneyer G, Kilian B, Graner A (2012) Genome-wide association studies for agronomical traits in a world wide spring barley collection. BMC Plant Biol 12

Pegadaraju V, Knepper C, Reese J, Shah J (2005) Premature leaf senescence modulated by the Arabidopsis PHYTOALEXIN DEFICIENT4 gene is associated with defense against the phloemfeeding green peach aphid. Plant Physiol 139:1927-1934

Pineda A, Zheng S-J, van Loon JJ, Pieterse CM, Dicke M (2010) Helping plants to deal with insects: the role of beneficial soil-borne microbes. Trends Plant Sci 15:507-514

Powell G, Pirone T, Hardie J (1995) Aphid stylet activities during potyvirus acquisition from plants and an in vitro system that correlate with subsequent transmission. Europ J Plant Pathol 101:313-321

Pozo MJ, Van Der Ent S, Van Loon L, Pieterse CM (2008) Transcription factor MYC2 is involved in priming for enhanced defense during rhizobacteria-induced systemic resistance in Arabidopsis thaliana. New Phytol 180:511-523

Prado E, Tjallingii WF (1994) Aphid activities during sieve element punctures. Ent Exp Appl 72:157-165

Rashid MH, Khan A, Hossain MT, Chung YR (2017) Induction of Systemic Resistance against Aphids by Endophytic Bacillus velezensis YC7010 via Expressing PHYTOALEXIN DEFICIENT4 in Arabidopsis. Front Plant Sci 8:211

Schenk ST, Schikora A (2015) AHL-priming functions via oxylipin and salicylic acid. Front Plant Sci 5:784

Schikora A, Schenk ST, Hartmann A (2016) Beneficial effects of bacteria-plant communication based on quorum sensing molecules of the $\mathrm{N}$-acyl homoserine lactone group. Plant Mol Biol 90:605-612

Schikora A, Schenk ST, Stein E, Molitor A, Zuccaro A, Kogel K-H (2011) N-acyl-homoserine lactone confers resistance toward biotrophic and hemibiotrophic pathogens via altered activation of AtMPK6. Plant Physiol 157:1407-1418

Schliephake E, Habekuss A, Scholz M, Ordon F (2013) Barley yellow dwarf virus transmission and feeding behaviour of Rhopalosiphum padi on Hordeum bulbosum clones. Ent Exp Appl 146:347-356

Schuhegger R, Ihring A, Gantner S, Bahnweg G, Knappe C, Vogg G, Hutzler P, Schmid M, Van Breusegem F, Eberl L (2006) Induction of systemic resistance in tomato by N-acyl-L-homoserine lactoneproducing rhizosphere bacteria. Plant Cell Environ 29:909-918 
Serteyn L, Quaghebeur C, Ongena M, Cabrera N, Barrera A, MolinaMontenegro MA, Francis F, Ramírez CC (2020) Induced systemic resistance by a plant growth-promoting rhizobacterium impacts development and feeding behavior of aphids. Insects 11:234

Shrestha A, Elhady A, Adss S, Wehner G, Böttcher C, Heuer H, Ordon F, Schikora A (2019) Genetic differences in barley govern the responsiveness to $\mathrm{N}$-acyl homoserine lactone. Phytobiomes Journal 3:191-202

Shrestha A, Schikora A (2020) AHL-priming for enhanced resistance as a tool in sustainable agriculture. FEMS Microbiol Ecol. https ://doi.org/10.1093/femsec/fiaa226

Shrestha A, Grimm M, Ojiro I, Krumwiede J, Schikora A (2020) Impact of quorum sensing molecules on plant growth and immune system. Front Microbiol 11:1545

Stenberg JA (2017) A conceptual framework for integrated pest management. Trends Plant Sci 22:759-769

Sternberg ED, Thomas MB (2017) Insights from agriculture for the management of insecticide resistance in disease vectors. Evol Appl 11:404-414

Sugio A, Dubreuil G, Giron D, Simon J-C (2015) Plant-insect interactions under bacterial influence: ecological implications and underlying mechanisms. J Exp Bot 66:467-478

Tétard-Jones C, Kertesz Michael A, Gallois P, Preziosi Richard F (2007) Genotype-by-genotype interactions modified by a third species in a plant-insect system. Am Nat 170:492-499

Tjallingii WF (1985) Electrical nature of recorded signals during stylet penetration by aphids. Ent Exp Appl 38:177-186

Tjallingii WF, Esch TH (1993) Fine-structure of aphid stylet routes in plant-tissues in correlation with EPG signals. Physiol Entomol 18:317-328

Van der Ent S, Van Hulten M, Pozo MJ, Czechowski T, Udvardi MK, Pieterse CM, Ton J (2009) Priming of plant innate immunity by rhizobacteria and $\beta$-aminobutyric acid: differences and similarities in regulation. New Phytol 183:419-431
Viswanath G, Sekar J, Prabavathy V (2016) Acyl homoserine lactoneproducing rhizobacteria elicit systemic resistance in plants. In: Choudhary DK, Varma A (eds) Microbial-mediated Induced Systemic Resistance in Plants. Springer, Singapore, pp 135-146

Wehner G, Kopahnke D, Richter K, Kecke S, Schikora A, Ordon F (2019) Priming is a suitable strategy to enhance resistance towards leaf rust in barley. J Phytobiomes 3:46-51

Wilkinson T (1998) The elimination of intracellular microorganisms from insects: an analysis of antibiotic-treatment in the pea aphid (Acyrthosiphon pisum). Comp Biochem Physiol A Mol Integr Physiol 119:871-881

Will T, Furch ACU, Zimmermann MR (2013) How phloem-feeding insects face the challenge of phloem-located defenses. Front Plant Sci 4:336

Zarkani AA, Stein E, Röhrich CR, Schikora M, Evguenieva-Hackenberg E, Degenkolb T, Vilcinskas A, Klug G, Kogel K-H, Schikora A (2013) Homoserine lactones influence the reaction of plants to rhizobia. Int J Mol Sci 14:17122-17146

Zhao Q, Zhang C, Jia Z, Huang Y, Li H, Song S (2015) Involvement of calmodulin in regulation of primary root elongation by $\mathrm{N}-3$-oxohexanoyl homoserine lactone in Arabidopsis thaliana. Front Plant Sci 5:807

Zimmerli L, Jakab G, Métraux J-P, Mauch-Mani B (2000) Potentiation of pathogen-specific defense mechanisms in Arabidopsis by $\beta$-aminobutyric acid. PNAS 97:12920-12925

Publisher's Note Springer Nature remains neutral with regard to jurisdictional claims in published maps and institutional affiliations. 